\title{
ASSOCIATION BETWEEN COMPUTER GAME TYPE, PLAYING TIME AND SENSE OF COHERENCE IN LITHUANIAN ADOLESCENTS
}

\author{
Ruta Ustinavičienė, Lina Škėmienė, Dalia Lukšienė, Ričardas Radišauskas, Gintarè Kalinienė, Paulius \\ Vasilavičius \\ Department of Environmental and Occupational Medicine, Lithuanian University of Health Sciences, Kaunas, Lithuania
}

\begin{abstract}
SUMMARY
Objective: As an excessive gaming is the addiction of the 21th century, purpose of our study was to investigate how sense of coherence interacts with gaming time, choice of various games types, age and gender characteristics. Our task was to evaluate relationship between computer gaming and an adolescent's sense of coherence.

Methods: A cross-sectional study based on a self-reported questionnaire was performed among the representative samples of 1,806 adolescents aged 13-18 years. Adolescents reported the amount of time spent gaming computer games, types of games, as well as sense of coherence. The relations between the choice of computer games type, time spent gaming computer games and sense of coherence of respondents were assessed by odds ratio using multivariate regression analysis.

Results: $32.9 \%$ of boys and $81.1 \%$ of girls reported gaming computer games less than 5 hours per day during the last month ( $p<0.001) .30 .9 \%$ of boys and $4.1 \%$ of girls reported gaming computer games more than 10 hours per day during the last month $(p<0.001)$. Boys and girls aged 13-15 with a weak sense of coherence had significantly higher probability to play action or combat computer games for 5 or more hours per day in comparison to the respondents who had a strong sense of coherence. Also, this probability was 2 times higher among boys than among girls.

Conclusion: Sense of coherence is a useful tool to identify adolescents who are at risk of excessive gaming. Game playing time is linked to the sense of coherence. Adolescent with a weak sense of coherence had a higher probability to play more times, especially in younger age.
\end{abstract}

Key words: computer games, sense of coherence, adolescent, pathological gaming, Lithuania

Address for correspondence: R. Ustinavičienè, Department of Environmental and Occupational Medicine, Public Health Faculty, Medical Academy, Lithuanian University of Health Sciences, 18 Tilzes str., 47181 Kaunas, Lithuania. E-mail: ruta.ustinaviciene@Ismuni.It

https://doi.org/10.21101/cejph.a4731

\section{INTRODUCTION}

Computers and the internet are an integral part of modern life. Television and computer games take up a large part of children's time (1-3). The internet addiction of adolescents and a long time spent playing games are related with family, social and psychological environment (3). Researchers have shown that people with lower self-esteem are more likely to become addicted to online gaming (4). Some studies have indicated that computer and video game consumption is associated with psychiatric symptoms, such as aggressive behaviour and hyperactivity disorder $(1,2)$.

Many studies analyse the influence of harmful health behaviour such as use of alcohol, smoking and overweight on health outcomes of adolescents $(5,6)$. Now, recent studies have shown that excessive gaming and internet addiction create new harmful health behaviour in adolescents $(7,8)$.

Studies reveal the possibility that some personal factors may play a key role in the development of adolescent internet addiction and excessive gaming $(9,10)$. One of the most popular theories that combine a number of personal factors is
Antonovsky's concept of sense of coherence (SOC). Antonovsky viewed SOC as a dispositional orientation or a coping resource which reflects a person's capacity to respond to stressful situations and life events (11). Data from a study representing Finnish 15 -year-old adolescents show that people with strong SOC may feel more in control of their lives, enjoy life more, and obtain better social support. Also, they tend to adopt a more positive and optimistic outlook of life, have higher self-esteem and deal successfully with stressors (12). At the same time, adolescents with weak SOC may easily adopt poor health behaviour, such as smoking, drinking of alcohol, lack of physical exercise, gain of overweight or poor oral health behaviour, involving diet or hygiene $(12,13)$.

As an excessive gaming is the addiction of the 21 th century ( 7 , 14), it is important to investigate how SOC interacts with gaming time, choice of games type, age and gender characteristics. There is currently a lack of research investigating the role of SOC in relation to types of computer games and gaming time. The purpose of the study is to evaluate relationship between computer gaming and an adolescent's sense of coherence. 


\section{MATERIALS AND METHODS}

\section{Sample and Setting}

The study design was cross-sectional. A multilevel sampling method (county, school and class) (15) was employed in composing a nationally representative sample in Lithuania (Kaunas County). Among Kaunas County inhabitants 94\% were of Lithuanian nationality, $98 \%$ of students attended municipality schools in Kaunas County. According to the Ministry of Education and Science data there are 56 municipality schools with 14,589 students from grade 9 to grade 11 in Kaunas County. Sample power analysis revealed that determined sample size was 1,450 students. In our survey, we randomly selected 20 schools out of 56 . From 240 classes in the selected schools, a random sample of 80 classes (every third) was selected. Thus 1,806 registered students from grade 9 to grade 11 (aged 13-18) were invited to participate in this study. The survey response rate was $95.8 \%(n=1,730)$. Information from 76 students has not been included in the survey mainly due to school children being absent from school when their class completed the survey or due to the fact that less than half of the questionnaire questions had been completed. The sample consisted of $50.2 \%(n=869)$ boys and $49.8 \%(n=861)$ girls. The mean age of male and female respondents was $15.86 \pm 0.94$ and $15.81 \pm 0.93$, respectively.

\section{Data Collection}

The questionnaire was distributed by researcher in selected schools during 2012. The students had the option to decline participation in the survey. The researcher did not attempt to persuade any of the potential respondents to participate.

The survey data included information about what grade and school each student attended, which made it possible to conclude that the absent students were randomly distributed between the schools. The survey was conducted with voluntary and anonymous participation of respondents.

\section{Questionnaire}

Self-report questionnaires were used in this study. The questionnaire consisted of four questions regarding socio-demographic data (gender, age, school, and grade); 13 items regarding sense of coherence and 5 questions regarding habits related to choice of computer game type and time spent playing computer games. In order to ascertain the period of time that respondents had spent playing computer games, students were asked "How many hours a day did you play computer games during the last month?" In order to identify the heavy computer games players, based on publications we have provided the following response options: $<5$ hours and $\geq 5$ hours (16). The respondents who played computer games were allowed to identify one of their most favourite computer games which they usually play. The most popular online games in the sample were: World of War Craft, World of Tanks, Grand Theft Auto, Quick Fire Pool, etc. According to the respondent's answers about their most favourite computer game, the students were classified in two groups: students with a preference for logic computer games, and students with a preference for action or combat computer games.

The evaluation of SOC was conducted by applying the short 13-item version of the SOC questionnaire by A. Antonovsky (17). The questionnaire was validated and adopted for the Lithuanian adolescent population (18). Research data showed, that the SOC13 questionnaire was the most frequently used version (in 54\% of reviewed studies) and had a good reliability (with an average Cronbach's alpha of 0.82). Each question/statement had a scale of 7 response options - from 1 (very frequently as the worst option) to 7 (never as the best option). The SOC-13 version sums responses to all 13 questions/statements, the total score ranges between 13 and 91, and the higher sum shows the better sense of coherence. For the evaluation of the respondents' sense of coherence, the subjects were distributed into three groups (tertile) (Table 1). The SOC tertile values for boys and girls did not differ significantly.

\section{Statistical Analysis}

Statistical analysis of data was performed using the software package SPSS 13.0 for Windows. The data were analysed by assessing the differences between gender, age, and the amount of time spent playing computer games with respect to the respondents' sense of coherence. The Z test was used to evaluate statistical hypotheses on the difference in the distribution of variables between the groups of respondents. The odds ratio (OR) of longer ( 5 or more hours per day) computer games play among boys and girls with respect to their sense of coherence in relation to age and type of computer games were assessed using logistic regression analysis. The level of statistical significance was set at $\mathrm{p}<0.05$.

\section{RESULTS}

The sample consisted of 869 (50.2\%) boys and 861 (49.8\%) girls. The age of respondents ranged from 13 to 18 years. Demo-

Table 1. Tertile margins of the sense of coherence $(N=1,730)$

\begin{tabular}{|c|c|c|c|c|}
\hline \multirow{2}{*}{ Sense of coherence tertiles } & \multirow{2}{*}{ Number $(\%)$ of respondents } & \multicolumn{3}{|c|}{ Tertile margins } \\
\hline & & Minimum & Maximum & Mean \pm SD \\
\hline $\begin{array}{l}\text { Tertile } 1 \\
\text { Weak sense of coherence }\end{array}$ & $581(33.6)$ & 22 & 55 & $48.98 \pm 5.49$ \\
\hline $\begin{array}{l}\text { Tertile } 2 \\
\text { Moderate sense of coherence }\end{array}$ & $602(34.8)$ & 56 & 64 & $59.88 \pm 2.41$ \\
\hline $\begin{array}{l}\text { Tertile } 3 \\
\text { Strong sense of coherence }\end{array}$ & $547(31.6)$ & 65 & 87 & $71.11 \pm 5.04$ \\
\hline
\end{tabular}


graphic characteristics of the respondents according to gender and age are shown in Table 2. Only $1.2 \%$ boys and $9.9 \%$ girls aged $13-18$ years did not play any computer games $(p<0.001)$. The majority of boys $(86.3 \%)$ in all age groups reported that they had played action or combat computer games more frequently than girls $(43.6 \%, \mathrm{p}<0.05)$. While girls played logic computer games about 4 times more frequently in comparison to boys, $46.6 \%$ and $12.5 \%(\mathrm{p}<0.05)$, respectively. The younger girls $(13-15$ years old) played action and combat computer games more frequently in comparison to older $(16-18$ years old $)$ girls $(\mathrm{p}<0.05)$.

The distribution of boys and girls playing different types of computer games by age and SOC is shown in Tables 3 and 4 .
Significant association between selected type of computer game and SOC was found only among younger girls (13-15 years old). Younger girls with a weak SOC reported that they played action or combat computer games more frequently than girls with strong SOC $(p=0.004)$. On the contrary, 13-15 years old girls with strong SOC significantly reported that they played logic computer games more frequently than girls with weak SOC $(p=0.0008)$ and with moderate SOC $(p=0.049)$. We analysed the association between the time per day that respondents had played computer games during the last month and SOC by gender, age and type of computer game (Table 5). We did not find significant associations between the probability of longer $(\geq 5$

Table 2. Characteristics of respondents according to gender and age $(N=1,730)$

\begin{tabular}{|c|c|c|c|c|}
\hline \multirow{2}{*}{ Attributes } & \multicolumn{2}{|c|}{$13-15$ years } & \multicolumn{2}{|c|}{$16-18$ years } \\
\hline & Boys & Girls & Boys & Girls \\
\hline Number of cases, $n$ & 315 & 356 & 554 & 505 \\
\hline Did not play any computer games, $\mathrm{n}(\%)$ & 0 & $29(8.1)$ & $10(1.8)$ & $56(11.1)^{\#}$ \\
\hline Played logic computer games, $n(\%)$ & $38(12.1)$ & $152(42.7)$ & $71(12.8)$ & $249(49.3)^{\#}$ \\
\hline Played action or combat computer games, $\mathrm{n}(\%)$ & $277(87.9)$ & $175(49.2)^{\#}$ & $473(85.4)$ & $200(39.6)^{\#}$ \\
\hline \multicolumn{5}{|c|}{ Time spent gaming logic computer games per day during last month, $n(\%)$} \\
\hline$<5$ hours & $20(52.6)$ & $132(86.8)^{\#}$ & $35(49.3)$ & $218(87.6)^{\#}$ \\
\hline$\geq 5$ hours & $18(47.4)$ & $20(13.2)^{\#}$ & $36(50.7)$ & $31(12.4)^{\#}$ \\
\hline \multicolumn{5}{|c|}{ Time spent gaming action or combat computer games per day during last month, $\mathrm{n}(\%)$} \\
\hline$<5$ hours & $90(32.5)$ & $127(72.6)^{\#}$ & $138(29.2)$ & $152(76.0)^{\#}$ \\
\hline$\geq 5$ hours & $187(67.5)$ & $48(27.4)^{\#}$ & $335(70.8)$ & $48(24.0)^{\#}$ \\
\hline
\end{tabular}

$\# p<0.001$ in comparison to boys in the same age group

Table 3. Distribution of boys playing different types of computer games according to sense of coherence and age $(N=869)$

\begin{tabular}{|l|c|c|c|c|c|}
\hline \multirow{2}{*}{ Age group } & $\begin{array}{c}\text { Sense of coherence } \\
\text { in tertiles }\end{array}$ & $\begin{array}{c}\text { Did not play any } \\
\text { computer games } \\
\mathbf{n}(\%)\end{array}$ & $\begin{array}{c}\text { Played logic computer } \\
\text { games } \\
\mathbf{n}(\%)\end{array}$ & $\begin{array}{c}\text { Played action or com- } \\
\text { bat computer games } \\
\mathbf{n}(\%)\end{array}$ & $\begin{array}{c}\text { Total } \\
\mathbf{n}(\%)\end{array}$ \\
\hline \multirow{3}{*}{$13-15$ years } & Strong & $0(0.0)$ & $18(17.1)$ & $87(82.9)$ & $105(33.3)$ \\
\cline { 2 - 6 } & Moderate & $0(0.0)$ & $8(8.3)$ & $88(91.7)$ & $96(30.5)$ \\
\cline { 2 - 6 } & Weak & $0(0.0)$ & $12(10.5)$ & $102(89.5)$ & $114(36.2)$ \\
\cline { 2 - 6 } & Strong & $4(2.2)$ & $23(12.7)$ & $154(85.1)$ & $181(32.7)$ \\
\cline { 2 - 6 } $16-18$ years & Moderate & $2(1.0)$ & $30(15.5)$ & $162(83.5)$ & $194(35.0)$ \\
\hline
\end{tabular}

Table 4. Distribution of girls playing different types of computer games according to sense of coherence and age $(N=861)$

\begin{tabular}{|l|c|c|c|c|c|}
\hline \multirow{2}{*}{ Age group } & $\begin{array}{c}\text { Sense of coherence } \\
\text { in tertiles }\end{array}$ & $\begin{array}{c}\text { Did not play any } \\
\text { computer games } \\
\mathbf{n}(\%)\end{array}$ & $\begin{array}{c}\text { Played logic computer } \\
\text { games } \\
\mathbf{n}(\%)\end{array}$ & $\begin{array}{c}\text { Played action or com- } \\
\text { bat computer games } \\
\mathbf{n}(\%)\end{array}$ & $\begin{array}{c}\text { Total } \\
\mathbf{n}(\%)\end{array}$ \\
\hline \multirow{3}{*}{$13-15$ years } & Strong & $7(6.7)$ & $57(54.8)$ & $40(38.5)$ & $104(29.2)$ \\
\cline { 2 - 6 } & Moderate & $11(7.9)$ & $59(42.1)^{*}$ & $70(50.0)$ & $140(39.3)$ \\
\cline { 2 - 6 } & Weak & $11(9.8)$ & $36(32.1)^{* * *}$ & $65(58.1)^{* *}$ & $112(31.5)$ \\
\hline \multirow{3}{*}{$16-18$ years } & Strong & $19(12.1)$ & $83(52.9)$ & $55(35.0)$ & $157(31.1)$ \\
\cline { 2 - 6 } & Moderate & $20(11.6)$ & $80(46.5)$ & $72(41.9)$ & $172(34.1)$ \\
\cline { 2 - 6 } & Weak & $17(9.6)$ & $86(48.9)$ & $73(41.5)$ & $176(34.8)$ \\
\hline
\end{tabular}

${ }^{*} p=0.0495$ ( $z$ test for two proportions) in comparison to $13-15$ years old girls with strong sense of coherence who played logic computer games; ${ }^{* *} p=0.004$ ( $z$ test for two proportions) in comparison to $13-15$ years old girls with strong sense of coherence who played action or combat computer games; ${ }^{* * *} p=0.0008$ 
hours) playing of logic computer games among boys and girls (irrespective of age) and their SOC (Table 5). However, logistic regression analysis showed that younger boys (13-15 years old) with a moderate SOC and a weak SOC had a higher probability to play action or combat computer games for 5 or more hours per day in comparison to boys who had a strong $\mathrm{SOC}(\mathrm{OR}=2.27$ and $\mathrm{OR}=2.49$, respectively). Younger girls with a weak $\mathrm{SOC}$ also had a higher probability to play action or combat computer games for 5 or more hours per day in comparison to girls who had a strong $\mathrm{SOC}(\mathrm{OR}=3.10)$.

Comparison of the findings between boys and girls aged 13-15 years showed that the boys with a weak SOC had a higher probability to play action or combat computer games for 5 or more hours per day in comparison to the girls of the same age with a weak SOC: $\mathrm{OR}=2.70(95 \%$ CI $1.61-4.53 ; \mathrm{p}<0.001)$. Also, the boys with a moderate SOC had a significantly higher probability to play action or combat computer games 5 or more hours per day in comparison to the girls with a moderate $\mathrm{SOC}: \mathrm{OR}=2.16$ (95\% CI 1.28-3.65; $\mathrm{p}=0.004)$.

\section{DISCUSSION}

Computer use and computer gaming have become an ever increasing part of many adolescents' daily lives $(7,19)$. Adolescents usually have less self-control, less self-regulation, lower social competence, so they are considered as the most vulnerable group to the temptations of computer games $(20,21)$. Computer games are divided into several categories: logic, action and combat. The choice of a game type is associated with gender: boys play combat or action games most frequently (22). This was confirmed by our investigation: combat and action games were chosen by boys more frequently, while girls usually choose logic games. According to the international research, adolescents usually choose action and combat games $(22,23)$. In our investigation younger girls played action and combat computer games more frequently in comparison to the oldest girls.

Researchers classify the duration spent playing computer games differently. Usually those, who spend 5 or more hours playing computer and video games, are considered as addicted to the computer $(16,24,25)$. There is no significant relation between playing computer games and age of the respondent, but it is established that boys play computer games more often and for longer periods of time than girls. Pathological involvement with computer games seems most frequently observed among adolescent boys $(16,22,25)$. Our study contrary to other similar findings showed that almost a half (48\%) of girls played action and combat games, but we also confirmed the fact that they spent less time for it in comparison with boys. Pathological gaming among adolescent girls actually predicts a decrease of aggression $(23,24)$. On the other hand, gaming increases aggression among boys. High gaming addiction is associated with aggression, low sociability and self-efficacy, and lower satisfaction with life $(1,26)$. Some research studies show that online game playing may induce physiological effects and influence on the physical mechanisms: playing violent games causes significantly higher sympathetic activity and diastolic blood pressure than playing nonviolent games (27).

Higher levels of pathological gaming, regardless of violent content, predict an increase in physical aggression among boys. Despite this effect, it is only applied to boys and does not diminish its importance, because adolescent boys are generally the heaviest players of violent games and most susceptible to pathological involvement (24). Data from other investigators confirm that the younger the computer games player the more time spent playing computer games (21). This can be explained taking into consideration that in the age of 16-18 people are more active, responsible for their future, and have more responsibilities. Long gaming and excessive use of internet can cause academic, social and interpersonal problems. It should be considered that

Table 5. The odds of longer (5 or more hours per day) computer games play among boys and girls with respect to their sense of coherence in relation to age and type of computer games

\begin{tabular}{|c|c|c|c|c|}
\hline \multirow{3}{*}{ Sense of coherence } & \multicolumn{2}{|c|}{ Boys } & \multicolumn{2}{|c|}{ Girls } \\
\hline & $13-15$ years & $16-18$ years & $13-15$ years & $16-18$ years \\
\hline & OR $(95 \% \mathrm{Cl})$ & $\mathrm{OR}(95 \% \mathrm{Cl})$ & OR $(95 \% \mathrm{Cl})$ & OR $(95 \% \mathrm{Cl})$ \\
\hline \multicolumn{5}{|l|}{ Logic computer games } \\
\hline Strong & 1 & 1 & 1 & 1 \\
\hline \multirow{2}{*}{ Moderate } & $1.12(0.26-4.91)$ & $0.55(0.15-1.96)$ & $1.06(0.35-3.23)$ & $0.55(0.22-1.39)$ \\
\hline & $p=0.880$ & $p=0.352$ & $p=0.913$ & $p=0.204$ \\
\hline \multirow{2}{*}{ Weak } & $2.33(0.37-14.6)$ & $0.33(0.10-1.13)$ & $0.36(0.10-1.39)$ & $0.65(0.27-1.60)$ \\
\hline & $p=0.365$ & $p=0.078$ & $p=0.139$ & $p=0.351$ \\
\hline \multicolumn{5}{|c|}{ Action or combat computer games } \\
\hline Strong & 1 & 1 & 1 & 1 \\
\hline \multirow{2}{*}{ Moderate } & $2.27(1.21-4.26)$ & $1.43(0.87-2.33)$ & $2.11(0.77-5.83)$ & $2.11(0.84-5.26)$ \\
\hline & $p=0.011$ & $p=0.160$ & $p=0.149$ & $p=0.110$ \\
\hline \multirow{2}{*}{ Weak } & $2.49(1.35-4.59)$ & $1.02(0.64-1.66)$ & $3.10(1.14-8.49)$ & $2.37(0.96-5.86)$ \\
\hline & $p=0.004$ & $p=0.908$ & $p=0.027$ & $p=0.061$ \\
\hline
\end{tabular}

OR - odds ratio, $\mathrm{Cl}$ - confidence interval 
children's psychological predisposition and family environment might significantly influence their computer use behaviour (15). Adolescents with pathological gaming and internet use have lower self-esteem and are more socially separated. At the same time, they have significantly lower general health questionnaire scores (28). Computer gaming is associated with addictive behaviour such as smoking and alcohol consumption (16). There are a number of studies, which show that adolescents with strong SOC report significantly lighter use of alcohol, being a non-smoker, better care of oral health, and better social competence in comparison to others. Pupils who use alcohol and have a positive attitude to the use of drugs show lower SOC scores $(29,30)$. Respondents reporting strong SOC scores may feel more in control of their lives, enjoy life more and obtain better social support compared to those with weaker SOC scores (30).

The relation between SOC and computer game type showed significant differences only for girls aged 13-15 years. Girls with a weak SOC reported that they played action or combat computer games 1.5 times more frequently than girls with a strong SOC. Gender differences found in the study correspond with data of previous studies, which indicate that girls in some areas are found to adjust more negatively to stressors which may lead to psychological unstableness $(13,20,28)$. The present study showed that those with a weak SOC chose an escapist strategy for coping with stress that is computer games. SOC research shows that adolescents with a strong SOC can more easily cope with environmental stress $(29,31)$. The findings are in line with studies showing that a strong SOC is associated with positive mental health (13). As studies show, adolescents under 15 years have a weaker SOC $(12,13)$. Changes in SOC take place throughout all life, especially in adolescence (13). Results suggest that low scores on SOC questionnaire are strongly associated with pathological gaming among adolescents of this age group. SOC is a useful tool for identifying adolescents who are at risk of pathological involvement to computer games. Scientific publications reveal the importance of the salutogenic approach in developmental research of children and adolescents, and its potential for educational planning and prevention (32).

Prevention and regulation of gaming is largely based on the perception of gaming-related harm. This differ from one country to another. It is possible to introduce policies that regulate gaming, but prevention of problematic gaming would be based more on educational efforts than on restrictive measures (33).

Our findings could be useful for school nurses, school psychologists, public health specialists, and people, who work with adolescents in other settings. The main task for school health professionals is to identify the adolescents who are at great risk of excessive/additive gaming behaviour. There is important to identify the computer use time, screen time in adolescent encounters. Questionnaire and estimation online gamers groups can be used for this task. Prevention programmes can be designed at school, community or regional levels. At the same time, health promotion programme for adolescents, including peer group, family members and school staff can be developed. School personnel need to know about the factors associated with computer games playing such as weak SOC - for the designing health promotion and prevention programmes. According to literature data, for high prevalence of problematic online gaming in adolescents individual treatment is recommended (34).

\section{Limitations}

Major strengths of our study were the large sample size and high response rate. However, the study had some limitations of research design. The study type is cross-sectional. For this reason, our study could examine only associations between reported gaming and SOC scores at one point at a time, instead of drawing conclusions about the underlying development of reported behavioural patterns. It is not possible to show causal relationship because there are a number of possible factors which may influence associations and need to be investigated more deeply. A longitudinal design of the study would have strengthened the findings, allowed the assessment of changes over time, and this is our plan for future research.

Our research is based on the self-reported measures, so we must believe that our respondents conscientiously filled in the questionnaires and provided an accurate evaluation of their gaming and psychological assessment. The large size of the study group of our study could have reduced the influence of potential random error related to self-reporting.

Many of the aforementioned limitations could be addressed as future prospective studies that investigate questions about socioeconomic background, education of parents, and computer game playing in more detail, which include a variety of investigation methods to evaluate causal relationship for development of pathological gaming, factors influencing sense of coherence, changes in specific behavioural patterns over time from primary school to adolescence.

\section{CONCLUSIONS}

This cross-sectional study is the first one to report relation between sense of coherence and computer games in Lithuania. There is a proof that sense of coherence is a useful tool to identify adolescents who are at risk of excessive gaming. According to the study results, game playing time is linked to the sense of coherence. Adolescent with a weak sense of coherence had a higher probability to play more times, especially in younger age.

\section{Conflict of Interests}

None declared

\section{Ethical Approval}

The study protocol was approved by the Department of Kaunas Municipality Administration Education and Culture Education and Education Division (Protocol No. 35-2-703), and the Regional Ethical Committee of Biomedical Researches (Protocol No. BE-2-61). Parental consent was obtained from each participant of the study.

\section{REFERENCES}

1. Frölich J, Lehmkuhl G, Döpfner M. Computer games in childhood and adolescence: relations to addictive behaviour, ADHD, and aggression. Z Kinder Jugendpsychiatr Psychother. 2009;37(5):393-402. (In German.)

2. Festl R, Scharkow M, Quandt T. Problematic computer game use among adolescents, younger and older adults. Addiction. 2013;108(3):592-9.

3. Sinkkonen HM, Puhakka H, Meriläinen M. Internet use and addiction among Finnish adolescents (15-19 years). J Adolesc. 2014;37(2):123-31.

4. Wan CS, Chiou WB. Why are adolescents addicted to online gaming? An interview study in Taiwan. Cyberpsychol Behav. 2006 Dec;9(6):762-6. 
5. García-Moya I, Jiménez-Iglesias A, Moreno C. Sense of coherence and substance use in Spanish adolescents. Does the effect of SOC depend on patterns of substance use in their peer group? Adicciones. 2013;25(2):10917.

6. Casiano H, Kinley DJ, Katz LY, Chartier MJ, Sareen J. Media use and health outcomes in adolescents: findings from a nationally representative survey. J Can Acad Child Adolesc Psychiatry. 2012;21(4):296-301.

7. Tam P, Walter G. Problematic internet use in childhood and youth: evolution of a 21st century affliction. Australas Psychiatr. 2013;21(6):533-6.

8. Aboujaoude E. Problematic Internet use: an overview. World Psychiatry. 2010;9(2):85-90.

9. Hilgard J, Engelhardt CR, Bartholow BD. Individual differences in motives, preferences, and pathology in video games: the gaming attitudes, motives, and experiences scales (GAMES). Front Psychol. 2013;4:608. doi: 10.3389/fpsyg.2013.00608.

10. Müller KW, Koch A, Dickenhorst U, Beutel, ME, Duven E. Wölfling K. Addressing the question of disorder-specific risk factors of internet addiction: a comparison of personality traits in patients with addictive behaviors and comorbid internet addiction. Biomed Res Int. 2013;2013:546342. doi: $10.1155 / 2013 / 546342$

11. Antonovsky A. Unraveling the mystery of health: how people manage stress and stay well. San Francisco: Jossey-Bass; 1987.

12. Mattila ML, Rautava P, Honkinen PL, Ojanlatva A, Jaakkola S, Aromaa $\mathrm{M}$, et al. Sense of coherence and health behaviour in adolescence. Acta Paediatr. 2011 Dec;100(12):1590-5.

13. Moksnes UK, Espnes GA, Haugan G. Stress, sense of coherence and emotional symptoms in adolescents. Psychol Health. 2013;29(1):32-49.

14. Kuss DJ. Internet gaming addiction: current perspectives. Psychol Res Behav Manag. 2013;14(6):125-37.

15. Saifuddin A. Methods in sample surveys. The Johns Hopkins University; 2009.

16. Hellström C, Nilsson KW, Leppert J, Åslund C. Influences of motives to play and time spent gaming on the negative consequences of adolescent online computer gaming. Comput Human Behav. 2012;28(4):1379-87.

17. Antonovsky A. The structure and properties of the sense of coherence scale. Soc Sci Med. 1993;36(6):725-33.

18. Malinauskas R, Malinauskiene, V, Dumciene A, Sniras S. Basic social skills and sense of coherence among children at risk. Sveikatos Mokslai. 2008;(5):1935-8. (In Lithuanian.)

19. Batthyány D, Müller KW, Benker F, Wölfling K. Computer game playing: clinical characteristics of dependence and abuse among adolescents. Wien Klin Wochenschr. 2009;121(15-16):502-9. (In German.)

20. Rivera F, García-Moya I, Moreno C. Ramos P. Developmental contexts and sense of coherence in adolescence: a systematic review. J Health Psychol. 2013;18(6):800-12.
21. Griffiths MD, Davies MN, Chappell D. Online computer gaming: a comparison of adolescent and adult gamers. J Adolesc. 2004;27(1):87-96.

22. Griffiths MD, Davies MN, Chappell D. Demographic factors and playing variables in online computer gaming. Cyberpsychol Behav. 2004 Aug;7(4):479-87.

23. Möller I, Krahé B. Exposure to violent video games and aggression in German adolescents: a longitudinal analysis. Aggress Behav. 2009;35(1):7589.

24. Lemmens JS, Valkenburg PM, Peter J. The effects of pathological gaming on aggressive behaviour. J Youth Adolesc. 2011;40(1):38-47.

25. Mentzoni RA, Brunborg GS, Molde H, Myrseth H, Skouveroe KJ, Hetland J, et al. Problematic video game use: estimated prevalence and associations with mental and physical health. Cyberpsychol Behav Soc Netw. 2011;14(10):591-6.

26. Konijn EA, Bijvank MN, Bushman BJ. I wish I were a warrior: the role of wishful identification in the effects of violent video games on aggression in adolescent boys. Dev Psychol. 2007;43(4):1038-44.

27. Lin TC. Effects of gender and game type on autonomic nervous system physiological parameters in long-hour online game players. Cyberpsychol Behav Soc Netw. 2013;16(11):820-7.

28. Niemz K, Griffiths M, Banyard P. Prevalence of pathological internet use among university students and correlations with self-esteem, the General Health Questionnaire (GHQ), and disinhibition. Cyberpsychol Behav. 2005 Dec;8(6):562-70.

29. Myrin B, Lagerström M. Health behaviour and sense of coherence among pupils aged 14-15. Scand J Caring Sci. 2006;20(3):339-46.

30. Moksnes UK, Haugan G. Stressor experience negatively affects life satisfaction in adolescents: the positive role of sense of coherence. Qual Life Res. 2015;24(10):2473-81.

31. Darling CA, McWey LM, Howard SN, Olmstead SB. College student stress: the influence of interpersonal relationships on sense of coherence. Stress Health 2007; 23(4):215-29.

32. Idan O, Margalit M. The salutogenic orientation: children's sense of coherence and hopeful thinking in education of children and adolescents. Inovatsie u nastavi. 2011;24(4):5-18.

33. Starcevic V, Billieux J. Precise estimates of gaming-related harm should guide regulation of gaming. J Behav Addict. 2018 Sept 1;7(3):522-5.

34. Zajac K, Ginley MK, Chang R, Petry NM. Treatments for Internet gaming disorder and Internet addiction: A systematic review. Psychol Addict Behav. 2017;31(8):979-94.

Received February 17, 2016 Accepted in revised form September 7, 2018 